\title{
Noticing cigarette health warnings and support for new health warnings among non-smokers in China: findings from the International Tobacco Control project (ITC) China survey
}

Zejun Li ${ }^{1}$, Tara Elton-Marshall ${ }^{2,3,4}$, Geoffrey T. Fong ${ }^{5,6,7}$, Anne Chiew Kin Quah' ${ }^{5}$, Guoze Feng ${ }^{8}$, Yuan Jiang ${ }^{8}$ and Sara C. Hitchman 9,10,1 ${ }^{*}$

\begin{abstract}
Background: Health warnings labels (HWLs) have the potential to effectively communicate the health risks of smoking to smokers and non-smokers, and encourage smokers to quit. This study sought to examine whether non-smokers in China notice the current text-only HWLs and whether they support adding more health information and including pictures on HWLs.

Methods: Adult non-smokers ( $n=1324)$ were drawn from Wave 4 (September 2011-November 2012) of the International Tobacco Control (ITC) China Survey. The proportion of non-smokers who noticed the HWLs, and supported adding more health information and pictures to the HWLs was examined. Additionally, the relation between non-smokers' demographic characteristics, including whether they had a smoking partner, their number of smoking friends, and noticing the HWLs and support for adding health information and pictures was examined. Because the HWLs changed during the survey period (April 2012), differences between non-smokers who completed the survey before and after the change were examined.

Results: $12.2 \%$ reported they noticed the HWLs often in the last month. The multivariate model, adjusting for demographics showed that respondents with a smoking partner $(\mathrm{OR}=2.41,95 \% \mathrm{Cl} 1.42-4.13, p=0.001)$ noticed the HWLs more often. $64.8 \%$ of respondents agreed that the HWLs should have more information, and $80.2 \%$ supported including pictures. The multivariate model showed that non-smokers who completed the survey after the HWLs were implemented $(\mathrm{OR}=0.63,95 \% \mathrm{Cl} 0.40-0.99, p=0.04)$ were less likely to support adding more health information. The multivariate model showed a significant relation between having a smoking partner and supporting pictorial HWLs (OR $=2.03,95 \% \mathrm{Cl} 1.24-3.33, p=0.005)$.

Conclusions: The findings indicate that the Chinese HWLs are noticed by a minority of non-smokers and that non-smokers strongly support strengthening the Chinese warning labels with more health information and pictures. Additionally, because the HWLs are noticed more often by non-smokers with a smoking spouse/partner, HWLs could be used to communicate the dangers of smoking and secondhand smoke exposure to non-smokers.
\end{abstract}

Keywords: China, Cigarette health warning labels, Non-smokers, Smoking, Health promotion

\footnotetext{
* Correspondence: sara.hitchman@kcl.ac.uk

${ }^{9}$ Department of Addictions, Institute of Psychiatry, Psychology, and

Neuroscience, King's College London, London, England

${ }^{10}$ UK Centre for Tobacco and Alcohol Studies, Nottingham, UK

Full list of author information is available at the end of the article
} 


\section{Background}

Globally, smoking is the leading cause of preventable death [1]. In 2015, approximately $49.3 \%$ of males and $2.0 \%$ of females 15 years and older smoked in China [2]. Due to the high prevalence of smoking, approximately $72.4 \%$ of non-smokers in China were exposed to secondhand smoke at least weekly, with $38 \%$ reporting daily exposure [3]. This is cause for concern as secondhand smoke exposure can cause significant health problems including adult heart disease, and lung diseases in children [3-5].

To reduce the harms from smoking, China ratified the World Health Organization's (WHO) Framework Convention on Tobacco Control (FCTC) in 2005 and took steps to implement the FCTC [6]. In January 2009, China changed the text-based HWLs that appeared on the side of the pack and replaced them with HWLs that covered $30 \%$ of the front and back bottom of the package. The warnings messages included two sets of general warnings (e.g., one of the two sets: 'Smoking is harmful to your health' and 'Quitting smoking early is good for your health'). Interestingly, the HWLs appearing on the back were printed in English and were identical to the Chinese characters on the front (Fig. 1) [6]. In April 2012, the Chinese HWLs were changed again. The English text on the back was changed to Chinese characters and the minimum text font size was increased from $2.0 \mathrm{~mm}$ to $4.0 \mathrm{~mm}$ (Fig. 2) [7]. Although the current HWLs in China meet the FCTC minimum requirements, they don't meet the FCTC guidelines for HWLs, which suggest that HWLs cover at least $50 \%$ of the pack and use pictures. Large pictorial health warnings have been shown to be more effective than smaller text-only HWLs and are important for informing people about the health risks of smoking and encouraging smokers to quit [8-12].

A previous study of the HWLs in China and Malaysia showed that when the HWLs in China were changed from text-only to larger text-only HWLs the proportion of smokers who noticed HWLs 'often' increased from 41.6 to $44.7 \%$, while in Malaysia, where pictorial HWLs were introduced, smokers who noticed HWLs 'often' or 'very often' increased from 54.4 to $67.0 \%$ [8]. However, there have been no studies to date examining noticing HWLs among non-smokers in China. Thus, it is worth considering the impact of HWLs on non-smokers. HWLs may warn non-smokers about the health effects of smoking and secondhand smoke, the health risks of smoking among their smoking family and friends, and may deter smoking uptake. HWLs may be particularly important for non-smokers in China for three main reasons: (1) Chinese non-smokers have a high rate of exposure at home to secondhand smoke and need to be warned about its harms [3-5], (2) China is a collectivistic culture and it is possible that non-smoking family members or close others could be a powerful influence on encouraging their close others to quit [13, 14], and (3) the low rates of smoking among women need to be maintained and HWLs could warn women about the health risks of starting to smoke. Thus, the aim of this study was to examine: (1) whether non-smokers in China notice the HWLs on cigarettes, (2) whether nonsmokers with smoking spouses or partners and friends notice the warnings more often, and (3) whether nonsmokers support adding more information and pictures to the current text only HWLs.
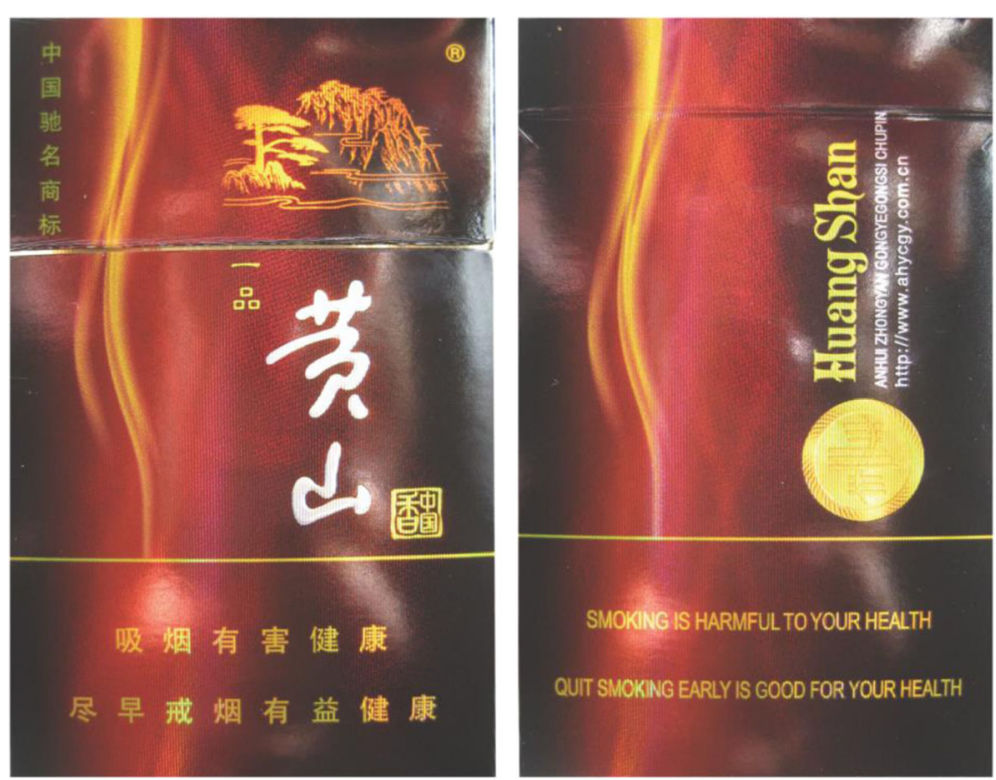

Fig. 12009 Chinese Health Warning Labels, left (front of pack), right (back of pack) 

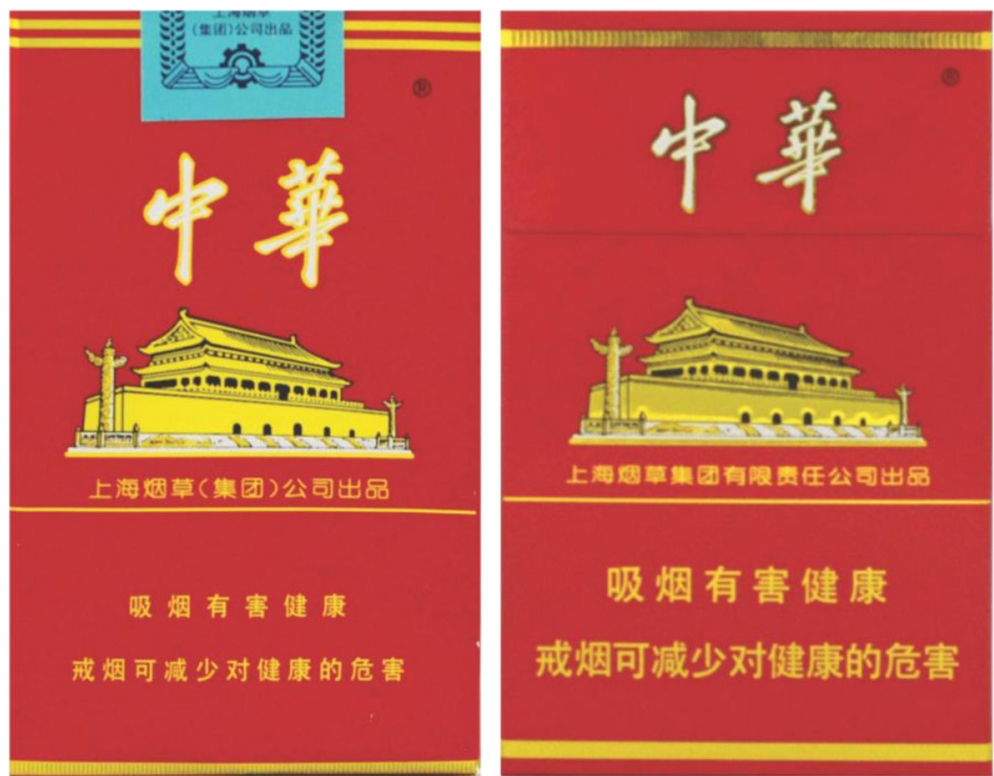

Fig. 2 Chinese Health Warning Labels, left (2009 warning labels), right (2012 larger warning labels)

\section{Methods}

\section{Survey}

Data are from the International Tobacco Control (ITC) China Survey Wave 4. The ITC China Survey is a cohort survey of adult smokers and non-smokers. The ITC China Survey used a multistage cluster sampling design to recruit 800 smokers and 200 non-smokers in each survey city: Stage 1 Jie Dao (street district), Stage $2 \mathrm{Ju}$ Wei Hui (residential blocks) and Stage 3 at the household level. The survey used quantitative methods and was conducted via face-to-face interviews in seven cities: Beijing, Shanghai, Changsha, Guangzhou, Yinchuan, Kunming, and Shenyang according to the geographical representation and levels of economic development difference [15]. Overall, there were 1324 non-smokers in the Wave 4 data collection. Further details about the methodology and survey design are available elsewhere $[15,16]$.

\section{Measures}

\section{Key outcome variables}

Noticing HWLs. 'In the last month, how often, if at all, have you noticed the health warnings on cigarette packages?' Responses were dichotomized as "often" ('often') vs. "less than often" ('once in a while,' 'never,' 'refused, or 'don't know.')

Support for more health information on HWLs. 'Do you think that cigarette packages should have more health information than they do now, less, or about the same amount as they do now?' Responses were dichotomized as "does not support" ('less information', 'same information' 'refused,' 'don't know') vs. "support" ('more health information').
Support for including pictures on HWLs. 'Would you support or oppose the government including pictures as part of the health warning on cigarette packs?' Responses were dichotomized as "does not support" ('strongly oppose, 'oppose,' 'refused,' 'don't know') vs. "support" ('strongly support', 'support').

Surveyed Before/After new HWLs implemented. Because the new HWLs were introduced while the survey was in the field, a variable was coded to indicate surveyed "before" vs. "after" the new HWLs were implemented.

\section{Key independent variables}

Partner and Friend Smoking Smoking spouse/partner. Respondents with a partner were asked: 'Does your partner or spouse smoke?' Responses were coded as "no smoking spouse/partner" = 'no'; "have smoking spouse/ partner" = 'yes'; "no spouse/partner" = 'refused', 'don't know'. Respondents who were not married or not living with their partners were coded as "no spouse/partner".

Number of smoking friends. 'Of the five closest friends or acquaintances (not including family members) that you spend time with on a regular basis, how many of them are smokers?' Responses were coded as “ $0,1,2,3,4,5$ ”.

\section{Demographic variables}

Demographic variables included: city, ethnicity (Han nationality, other nationality), age (18-39, 40-54 and 55+), gender (male, female), monthly household income (Low/ Medium: <¥3000, High: ¥¥3000), and education (Low/ Medium = no education, elementary school, junior/senior high school, High = college, university or higher. 


\section{Statistical analysis}

The data were analysed using Stata/MP 14.0 software for Mac. Descriptive and sample characteristics were examined. Bivariate and multiple logistic regression models (controlling for demographics) were used to examine the relation between the outcome, noticing the HWLs often vs. less often in the last month, and respondents' number of smoking friends, whether respondents had a smoking spouse/partner, and whether they were surveyed before vs. after new HWLs were introduced. To examine differences in support for adding more information and including pictures on Chinese HWLs, the predicted outcome of a logistic regression analysis was set to "support for more information" versus "no support" and "support for including pictures" versus "no support". These analyses were controlled for demographics, noticing HWLs in the last month, number of smoking friends, having a smoking partner and surveyed before versus after new HWLs were introduced. All analyses were conducted on weighted data.

\section{Results}

\section{Demographic characteristics}

The majority (92.7\%) of the respondents were Han, female (62.6\%), over the age of 55 (48.2\%), had high household income $(63.2 \%)$, and had low or medium education (67.5\%). 31.34\% had a smoking spouse/partner and most had no smoking friends (30.9\%). Very few respondents had 4 (5.2\%) or 5 (8.5\%) smoking friends (See Table 1$)$.

\section{Noticing HWLs}

$12.2 \%$ of respondents reported noticing HWLs often, $20.1 \%$ reported noticing HWLs 'once in a while' and $67.7 \%$ reported 'never' noticing HWLs in the last month. In the bivariate logistic regression analysis, non-smokers who had a smoking spouse/partner $(\mathrm{OR}=2.06,95 \% \mathrm{CI}$ 1.24-3.42 $p=0.006)$, with 5 vs. 0 smoking friends $(\mathrm{OR}=2.72,95 \%$ CI 1.09-6.82, $p=0.033)$, and surveyed after the new HWLs were implemented (OR = 2.09, 95\% CI $1.20-3.63, p=0.010$ ) were more likely to notice the HWLs more often. In multivariate logistic regression analysis, non-smokers who had a smoking spouse/partner $(\mathrm{OR}=2.41,95 \%$ CI 1.42-4.13, $p=0.002)$ noticed HWLs more often than those who did not have smoking spouse/ partner. Additional findings included, older and Han ethnicity non-smokers were less likely to notice the warnings. There were also unexpected city differences in noticing the HWLs (See Table 1).

\section{Support for strengthening the HWLs with more information and pictures}

About $64.8 \%$ of respondents agreed that cigarette packages should have more health information and $80.2 \%$ supported government including pictures as part of the
HWLs. In the bivariate logistic regression model, for non-smokers who supported adding more health information, there were no significant differences in whether they had a smoking spouse/partner or not, smoking friends and whether they were interviewed before or after the new HWLs were implemented. In the multivariate logistic regression analysis, non-smokers who were interviewed after the new HWLs were implemented (OR $=0.63,95 \%$ CI $0.40-0.99, p=0.04$ ) were less likely to support adding more health information (See Table 2). There were also city differences. In the bivariate logistic regression analysis, non-smokers who noticed HWLs in the last month $(\mathrm{OR}=2.52,95 \%$ CI $1.35-4.71, p=0.004)$ and who had smoking spouse $(\mathrm{OR}=2.12,95 \%$ CI $1.34-3.38, p=0.002)$ were more likely to support including pictures on the HWLs. In the multivariate logistic regression analyses, non-smokers who had a smoking spouse or partner $(\mathrm{OR}=2.03,95 \%$ CI $1.24-3.33, p=0.005)$ were more likely to support including pictures in HWLs, with no differences between those surveyed before or after the new HWLs were implemented (See Table 3). There were also city differences.

\section{Discussion}

In this study, we found that $12.2 \%$ of non-smokers noticed HWLs "often" in the last month. Respondents with a smoking spouse/partner and with 5 friends who smoke were more likely to notice the HWLs often. It is likely that non-smokers with smoking partners and friends had more opportunities to notice cigarette packs, and the HWLs in their daily life if their partner or friends smoked around them, and left cigarette packs out in the open. Although the proportion of respondents who noticed the HWLs was higher among those who completed the survey after the new HWLs were implemented (19.5\% after vs. $10.4 \%$ before), the overall proportion who noticed the HWLs was still very low. It is possible that this increase in noticing was due to both the size of the text being increased, removing the English warning, and also an initial novelty effect of seeing a new HWL [17].

Sixty-four percent of respondents supported adding more health information to the HWLs, and $80.2 \%$ supported including pictures as part of HWLs. Another study in four cities (Beijing, Shanghai, Kunming and Yinchuan) of China in 2010 showed that among 357 adult non-smokers, $77.5 \%$ supported adding more health information to the HWLs, and $86.1 \%$ of non-smokers supported including pictures as part of HWLs [6]. The result from this study is also consistent with another study based on data from Jiangsu province in 2011, that showed that most people thought the text-only HWLs did not provide useful health information about the risks of smoking and that the HWLs should have more health 
Table 1 Characteristics associated with whether non-smokers noticed health warning labels often vs. less than often in the last month $(N=1324)$

\begin{tabular}{|c|c|c|c|c|c|c|}
\hline & $N$ & $\%$ & $\begin{array}{l}\text { Noticed HWLs } \\
\text { Often (\%) }\end{array}$ & $p$ & OR & $95 \% \mathrm{Cl}$ \\
\hline \multicolumn{7}{|l|}{ City } \\
\hline Beijing & 202 & $15.25 \%$ & $3.49 \%$ & ref & ref & ref \\
\hline Shenyang & 187 & $14.12 \%$ & $8.16 \%$ & 0.192 & 2.45 & $0.63-9.52$ \\
\hline Shanghai & 196 & $14.80 \%$ & $3.75 \%$ & 0.831 & 1.17 & $0.28-4.87$ \\
\hline Changsha & 180 & $13.60 \%$ & $17.78 \%$ & $0.033^{\mathrm{a}}$ & 4.72 & $1.14-19.62$ \\
\hline Guangzhou & 191 & $14.43 \%$ & $11.31 \%$ & $0.015^{\mathrm{a}}$ & 4.07 & $1.33-12.46$ \\
\hline Kunming & 180 & $13.60 \%$ & $19.57 \%$ & $0.01^{\mathrm{a}}$ & 5.02 & $1.48-17.05$ \\
\hline Yinchuan & 188 & $14.20 \%$ & $23.04 \%$ & $0.002^{a}$ & 7.12 & $2.12-23.92$ \\
\hline \multicolumn{7}{|l|}{ Ethnicity } \\
\hline Others & 97 & $7.33 \%$ & $28.65 \%$ & ref & ref & ref \\
\hline Han nationality & 1227 & $92.67 \%$ & $11.08 \%$ & $0.007^{a}$ & 0.40 & $0.21-0.77$ \\
\hline \multicolumn{7}{|l|}{ Gender } \\
\hline Male & 495 & $37.39 \%$ & $12.34 \%$ & ref & ref & ref \\
\hline Female & 829 & $62.61 \%$ & $12.08 \%$ & 0.110 & 0.64 & $0.37-1.11$ \\
\hline \multicolumn{7}{|l|}{ Age (years) } \\
\hline $18-39$ & 248 & $18.73 \%$ & $21.27 \%$ & ref & ref & ref \\
\hline $40-54$ & 438 & $33.08 \%$ & $13.24 \%$ & 0.097 & 0.60 & $0.32-1.10$ \\
\hline $55+$ & 638 & $48.19 \%$ & $8.72 \%$ & $0.014^{a}$ & 0.48 & $0.27-0.86$ \\
\hline \multicolumn{7}{|c|}{ Household Income per month (yuan) } \\
\hline Low/Medium & 487 & $36.78 \%$ & $11.31 \%$ & ref & ref & ref \\
\hline High & 837 & $63.22 \%$ & $12.64 \%$ & 0.100 & 1.56 & $0.92-2.66$ \\
\hline \multicolumn{7}{|l|}{ Education } \\
\hline Low/Medium & 894 & $67.52 \%$ & $11.06 \%$ & & & \\
\hline High & 430 & $32.48 \%$ & $14.90 \%$ & 0.978 & 1.00 & $0.51-1.93$ \\
\hline \multicolumn{7}{|l|}{ Smoking spouse/partner } \\
\hline No smoking spouse/partner & 721 & $54.46 \%$ & $10.28 \%$ & ref & ref & ref \\
\hline Have smoking spouse/partner & 415 & $31.34 \%$ & $19.13 \%$ & $0.002^{\mathrm{a}}$ & 2.41 & $1.42-4.13$ \\
\hline No spouse/partner & 188 & $14.20 \%$ & $8.15 \%$ & 0.763 & 0.90 & $0.43-1.86$ \\
\hline \multicolumn{7}{|l|}{ Number of smoking friends } \\
\hline 0 & 409 & $30.89 \%$ & $10.57 \%$ & ref & ref & ref \\
\hline 1 & 238 & $17.98 \%$ & $10.35 \%$ & 0.617 & 0.80 & $0.33-1.95$ \\
\hline 2 & 264 & $19.94 \%$ & $10.12 \%$ & 0.204 & 0.60 & $0.27-1.33$ \\
\hline 3 & 232 & $17.52 \%$ & $13.72 \%$ & 0.821 & 0.92 & $0.46-1.84$ \\
\hline 4 & 69 & $5.21 \%$ & $9.74 \%$ & 0.647 & 0.69 & $0.14-3.39$ \\
\hline 5 & 112 & $8.46 \%$ & $24.35 \%$ & 0.333 & 1.59 & $0.62-4.11$ \\
\hline \multicolumn{7}{|c|}{ Surveyed before/after new HWLs apply } \\
\hline Before & 1100 & $83.08 \%$ & $10.40 \%$ & ref & ref & ref \\
\hline After & 224 & $16.92 \%$ & $19.50 \%$ & 0.628 & 1.17 & $0.61-2.25$ \\
\hline
\end{tabular}

Cl Confidence interval; Significant levels are indicated as follows: ${ }^{*} p<0.05 ;{ }^{* *} p<0.001,{ }^{* * *} p<0.0001$

OR, Odd Ratio of noticed health warning labels ( 0 , refused/don't know/once a while noting warning labels in last month; 1 , often noticed labels in last month) Survey before/after new HWLs apply, Before (before 01/04/2012); After (after 01/04/2012)

Notice HWLs often, Respondents who reported they notice HWLs often in the last month

${ }^{\text {a } T h e ~ p e r c e n t a g e ~ a r e ~ w e i g h t e d ~ a n d ~ t h e ~ f r e q u e n c i e s ~ a r e ~ u n w e i g h t e d ~}$ 
Table 2 Characteristics associated with whether non-smokers supported adding more information to cigarette packages $(N=1324)$

\begin{tabular}{|c|c|c|c|c|c|}
\hline & $N$ & Support more information (\%) & $p$ & OR & $95 \% \mathrm{Cl}$ \\
\hline \multicolumn{6}{|l|}{ City } \\
\hline Beijing & 202 & $69.78 \%$ & ref & ref & ref \\
\hline Shenyang & 187 & $66.02 \%$ & 0.797 & 0.94 & $0.57-1.54$ \\
\hline Shanghai & 196 & $55.93 \%$ & 0.111 & 0.49 & $0.20-1.18$ \\
\hline Changsha & 180 & $52.53 \%$ & $0.009^{\mathrm{a}}$ & 0.50 & $0.29-0.84$ \\
\hline Guangzhou & 191 & $64.88 \%$ & 0.266 & 0.75 & $0.44-1.25$ \\
\hline Kunming & 180 & $76.18 \%$ & 0.108 & 1.50 & $0.91-2.46$ \\
\hline Yinchuan & 188 & $69.79 \%$ & 0.413 & 1.28 & $0.70-2.34$ \\
\hline \multicolumn{6}{|l|}{ Ethnicity } \\
\hline Others & 97 & $62.51 \%$ & ref & ref & ref \\
\hline Han nationality & 1227 & $64.94 \%$ & 0.276 & 1.37 & $0.77-2.42$ \\
\hline \multicolumn{6}{|l|}{ Gender } \\
\hline Male & 495 & $62.54 \%$ & ref & ref & ref \\
\hline Female & 829 & $66.47 \%$ & 0.787 & 1.05 & $0.72-1.55$ \\
\hline \multicolumn{6}{|l|}{ Age (years) } \\
\hline $18-39$ & 248 & $72.57 \%$ & ref & ref & ref \\
\hline $40-54$ & 438 & $68.33 \%$ & 0.184 & 0.70 & $0.41-1.19$ \\
\hline $55+$ & 638 & $60.14 \%$ & $0.008^{\mathrm{a}}$ & 0.49 & $0.29-0.82$ \\
\hline \multicolumn{6}{|c|}{ Household Income per month (yuan) } \\
\hline Low/Medium & 487 & $63.41 \%$ & ref & ref & ref \\
\hline High & 837 & $65.49 \%$ & 0.292 & 1.21 & $0.84-1.75$ \\
\hline \multicolumn{6}{|l|}{ Education } \\
\hline Low/Medium & 894 & $64.58 \%$ & ref & ref & ref \\
\hline High & 430 & $65.28 \%$ & 0.209 & 0.80 & $0.57-1.13$ \\
\hline \multicolumn{6}{|l|}{ Smoking spouse/partner } \\
\hline No smoking spouse/partner & 721 & $63.76 \%$ & ref & ref & ref \\
\hline Have smoking spouse/partner & 415 & $67.06 \%$ & 0.799 & 1.07 & $0.65-1.75$ \\
\hline No spouse/partner & 188 & $65.22 \%$ & 0.895 & 1.04 & $0.61-1.75$ \\
\hline \multicolumn{6}{|c|}{ Noticed warning labels in last month } \\
\hline Less than Often & 1152 & $64.84 \%$ & ref & ref & ref \\
\hline Often & 172 & $64.35 \%$ & 0.581 & 0.89 & $0.58-1.36$ \\
\hline \multicolumn{6}{|l|}{ Number of smoking friends } \\
\hline 0 & 409 & $62.74 \%$ & ref & ref & ref \\
\hline 1 & 238 & $68.62 \%$ & 0.565 & 1.18 & $0.66-2.11$ \\
\hline 2 & 264 & $67.69 \%$ & 0.774 & 1.08 & $0.64-1.83$ \\
\hline 3 & 232 & $70.01 \%$ & 0.515 & 1.19 & $0.70-2.01$ \\
\hline 4 & 69 & $46.87 \%$ & 0.054 & 0.44 & $0.19-1.01$ \\
\hline 5 & 112 & $60.77 \%$ & 0.425 & 0.79 & $0.43-1.43$ \\
\hline \multicolumn{6}{|c|}{ Surveyed before/after new HWLs apply } \\
\hline Before & 1100 & $66.04 \%$ & & & \\
\hline After & 224 & $59.64 \%$ & $0.044^{\mathrm{a}}$ & 0.63 & $0.40-0.99$ \\
\hline
\end{tabular}

$\mathrm{Cl}$, Confidence interval; Significant levels are indicated as follows: ${ }^{*} p<0.05 ;{ }^{* *} p<0.001,{ }^{* * *} p<0.0001$

$\mathrm{OR}$, Odd Ratio of adding more health information ( 0 , refused/don't know/ less health information/the same; 1 , more health information)

Survey before/after new HWLs apply, Before (before 01/04/2012); After (after 01/04/2012)

${ }^{a}$ The percentage are weighted and the frequencies are unweighted 
Table 3 Characteristics associated with whether non-smokers reported they support government including pictures as part of the health warning labels $(N=1324)$

\begin{tabular}{|c|c|c|c|c|c|}
\hline & $N$ & Support pictures (\%) & $p$ & OR & $95 \% \mathrm{Cl}$ \\
\hline \multicolumn{6}{|l|}{ City } \\
\hline Beijing & 202 & $73.59 \%$ & ref & ref & ref \\
\hline Shenyang & 187 & $82.59 \%$ & 0.170 & 1.77 & $0.78-4.05$ \\
\hline Shanghai & 196 & $70.22 \%$ & 0.702 & 0.84 & $0.33-2.12$ \\
\hline Changsha & 180 & $90.86 \%$ & $0.013^{\mathrm{a}}$ & 3.22 & $1.28-8.07$ \\
\hline Guangzhou & 191 & $82.15 \%$ & 0.159 & 1.69 & $0.81-3.50$ \\
\hline Kunming & 180 & $87.05 \%$ & $0.039^{a}$ & 2.42 & $1.05-5.60$ \\
\hline Yinchuan & 188 & $76.07 \%$ & 0.704 & 1.14 & $0.58-2.25$ \\
\hline \multicolumn{6}{|l|}{ Ethnicity } \\
\hline Others & 97 & $75.46 \%$ & ref & ref & ref \\
\hline Han nationality & 1227 & $80.48 \%$ & 0.155 & 1.62 & $0.83-3.17$ \\
\hline \multicolumn{6}{|l|}{ Gender } \\
\hline Male & 495 & $78.65 \%$ & ref & ref & ref \\
\hline Female & 829 & $81.29 \%$ & 0.555 & 0.90 & $0.64-1.27$ \\
\hline \multicolumn{6}{|l|}{ Age (years) } \\
\hline $18-39$ & 248 & $86.67 \%$ & ref & ref & ref \\
\hline $40-54$ & 438 & $83.03 \%$ & 0.899 & 0.97 & $0.56-1.65$ \\
\hline $55+$ & 638 & $76.34 \%$ & 0.145 & 0.71 & $0.44-1.13$ \\
\hline \multicolumn{6}{|l|}{ Household Income per month (yuan) } \\
\hline Low/Medium & 487 & $80.19 \%$ & ref & ref & ref \\
\hline High & 837 & $80.14 \%$ & 0.440 & 1.19 & $0.77-1.84$ \\
\hline \multicolumn{6}{|l|}{ Education } \\
\hline Low/Medium & 894 & $79.40 \%$ & ref & ref & ref \\
\hline High & 430 & $81.99 \%$ & 0.767 & 0.94 & $0.64-1.39$ \\
\hline \multicolumn{6}{|l|}{ Smoking spouse/partner } \\
\hline No smoking spouse/partner & 721 & $77.46 \%$ & ref & ref & ref \\
\hline Have smoking spouse/partner & 415 & $87.97 \%$ & $0.005^{\mathrm{a}}$ & 2.03 & $1.24-3.33$ \\
\hline No spouse/partner & 188 & $78.07 \%$ & 0.793 & 1.07 & $1.04-3.86$ \\
\hline \multicolumn{6}{|c|}{ Noticed warning labels in last month } \\
\hline Less than Often & 1152 & $78.75 \%$ & ref & ref & ref \\
\hline Often & 172 & $90.33 \%$ & $0.039^{\mathrm{a}}$ & 2.00 & $1.04-3.86$ \\
\hline \multicolumn{6}{|l|}{ Number of smoking friends } \\
\hline 0 & 409 & $78.85 \%$ & ref & ref & ref \\
\hline 1 & 238 & $78.84 \%$ & 0.719 & 0.89 & $0.47-1.69$ \\
\hline 2 & 264 & $80.35 \%$ & 0.643 & 0.88 & $0.49-1.55$ \\
\hline 3 & 232 & $81.11 \%$ & 0.818 & 1.07 & $0.60-1.92$ \\
\hline 4 & 69 & $81.25 \%$ & 0.748 & 1.14 & $0.50-2.62$ \\
\hline 5 & 112 & $84.17 \%$ & 0.885 & 0.94 & $0.38-2.29$ \\
\hline \multicolumn{6}{|c|}{ Surveyed before/after new HWLs apply } \\
\hline Before & 1100 & $79.88 \%$ & ref & ref & ref \\
\hline After & 224 & $81.31 \%$ & 0.362 & 0.80 & $0.50-1.29$ \\
\hline
\end{tabular}

Cl, Confidence interval; Significant levels are indicated as follows: ${ }^{*} p<0.05 ;{ }^{* *} p<0.001$, ${ }^{* * *} p<0.0001$

OR, Odd Ratio of supporting government including pictures as part of health warnings ( 0 , refused/don't know/neither support nor oppose/oppose/strongly oppose; 1, support/strongly support)

Survey before/after new HWLs apply, Before (before 01/04/2012); After (after 01/04/2012)

Support for including pictures, Respondents who reported they support government should include pictures as part of health warning labels

${ }^{a}$ The percentage are weighted and the frequencies are unweighted 
information not only for smokers but also for nonsmokers [18].

The main strength of this study is its representative sample of non-smokers from seven Chinese cities, most current studies are among smokers. The main limitations are the self-reported measures allowing the possibility for social desirability effects, and the cross-sectional design. However, a longitudinal design to evaluate pictorial HWL in China is not possible unless pictorial HWLs are implemented.

\section{Conclusion}

The current HWLs in China are noticed by a minority of non-smokers and there is strong support to add more information and pictures to the HWLs. More effective HWLs could be particularly useful in China for educating non-smokers about the health risks of secondhand smoke. Additionally, educating non-smokers about the health risks of smoking with stronger HWLs may lead nonsmokers to encourage family/close others to quit, and help prevent smoking uptake [19]. Together, with findings from previous studies, the current study suggests that the Chinese HWLs should be strengthened to at least meet the FCTC guidelines of 50\% pictorial warnings. This study and others, from countries such as Canada, suggest that non-smokers strongly support including pictures as part of the HWLs, and that pictorial HWL more effectively communicate information about health risks [6, 8, 18-23].

\section{Abbreviations}

Cl: Confidence interval; HWLs: health warning labels; ITC: International tobacco control; OR: Odd ratio; WHO: World Health Organization

\section{Acknowledgements}

The authors thank Dr. Steve Xu, and Dr. Genevieve Sansone for their help with data access. The authors also thank the city teams for collecting the data.

\section{Funding}

The ITC China Project was supported by grants from the US National Cancer Institute at the National Institutes of Health (R01 CA125116), Canadian Institutes for Health Research (115016); and Chinese Center for Disease Control and Prevention. Additional support was provided to Geoffrey T. Fong from a Senior Investigator Award from the Ontario Institute for Cancer Research and a Prevention Scientist Award from the Canadian Cancer Society Research Institute. $\mathrm{SH}$ is a member of the UK Centre for Tobacco \& Alcohol Studies, a UK Clinical Research Collaboration Public Health Research: Centre of Excellence whose work is supported by funding from the Medical Research Council, British Heart Foundation, Cancer Research UK, Economic and Social Research Council, and the National Institute for Health Research under the auspices of the UK Clinical Research Collaboration (MR/K023195/1). The funding bodies played no role in the design of the study and collection, analysis, and interpretation of the data.

\section{Availability of data and materials}

The data that support the findings of this study are available from ITC China project but restrictions apply to the availability of these data, which were used under license for the current study, and so are not publicly available. Data are however available from the authors upon reasonable request and with permission of ITC China project.

\section{Authors' contributions}

$\mathrm{ZL}$ conducted the analyses, contributed to the design of the study, and led the writing of the manuscript. SCH provided major input on research design, analyses, and contributed to writing the manuscript. TEM provided input on research design and analyses, and was involved in the design and set-up of the ITC China Survey. GTF provided input on research design and analyses, and was involved in the design and set-up of the ITC China Survey. ACKQ provided input on research design and analyses, and was involved in the design and set-up of the ITC China Survey. GF was involved in the design and set-up of the ITC China Survey. YJ was involved in the design and set-up of the ITC China Survey. All authors reviewed the manuscript and provided critical input. All authors read and approved the final manuscript.

\section{Competing interests}

The authors declare that they have no competing interests.

\section{Consent for publication}

This manuscript contains no individual person's details, images, or videos.

\section{Ethics approval and consent to participate}

Ethical approval was obtained from the Office of Research at the University of Waterloo (Waterloo, Canada) and the Institutional Review Boards at the Roswell Park Cancer Institute (Buffalo, USA), the Cancer Council Victoria (Victoria, Australia), and the Chinese Center for Disease Control and Prevention (Beijing, China). Written consent to participate was obtained from all participants.

\section{Publisher's Note}

Springer Nature remains neutral with regard to jurisdictional claims in published maps and institutional affiliations.

\section{Author details}

${ }^{1}$ Department of Basic and Clinical Neuroscience, Institute of Psychiatry, Psychology, and Neuroscience, King's College London, London, England. ${ }^{2}$ Social and Epidemiological Research Department, Centre for Addiction and Mental Health, London, Canada. ${ }^{3}$ Department of Epidemiology and Biostatistics, Western University, London, Canada. ${ }^{4}$ Dalla Lana School of Public Health, University of Toronto, Toronto, Canada. ${ }^{5}$ Department of Psychology, University of Waterloo, Waterloo, Canada. ${ }^{6}$ School of Public Health and Health Systems, University of Waterloo, Waterloo, Canada. ${ }^{7}$ Ontario Institute for Cancer Research, Toronto, ON, Canada. ${ }^{8}$ Tobacco Control Office, Chinese Center for Disease Control and Prevention, Beijing, China. ${ }^{9}$ Department of Addictions, Institute of Psychiatry, Psychology, and Neuroscience, King's College London, London, England. ${ }^{10}$ UK Centre for Tobacco and Alcohol Studies, Nottingham, UK. ${ }^{11}$ Addictions Department, Addictions Sciences Building, Institute of Psychiatry, Psychology and Neuroscience (IoPPN), King's College London, 4 Windsor Walk, Denmark Hill, London SE5 8BB, England.

Received: 15 August 2016 Accepted: 8 May 2017

Published online: 19 May 2017

\section{References}

1. WHO report on the global tobacco epidemic, 2011. warning about the dangers of tobacco. Geneva: World Health Organization. 2011. [http://apps.who.int/iris/ bitstream/10665/44616/1/9789240687813_eng.pdf]. Accessed 3 May 2016.

2. WHO global report on trends in prevalence of tobacco smoking 2015 Geneva: World Health Organization; 2015. [http://apps.who.int/iris/bitstream/ 10665/156262/1/9789241564922_eng.pdf]. Accessed 3 May 2016.

3. Chinese Center for Disease Control and Prevention. Global adult tobacco survey (GATS) China 2010 Country report. Beijing: Chinese Centre for Disease Control and Prevention; 2011. http://www.who.int/tobacco/ surveillance/survey/gats/en_gats_china_report.pdf]. Accessed 3 May 2016

4. US Department of Health Human Services. How tobacco smoke causes disease: the biology and behavioral basis for smoking-attributable disease: a report of the Surgeon General. Atlanta, GA: US Department of Health and Human Services, Centers for Disease Control and Prevention, National Center for Chronic Disease Prevention and Health Promotion, Office on Smoking and Health. 2010. [http://www.ncbi.nlm.nih.gov/books/NBK53017/ pdf/Bookshelf_NBK53017.pdf]. Accessed 3 May 2016.

5. Öberg M, Woodward A, Jaakkola M, Peruga A, Prüss-Üstün A. Global estimate of the burden of disease from second-hand smoke. Geneva: World Health Organization; 2010. http://apps.who.int/iris/bitstream/10665/44426/1/ 9789241564076_eng.pdf]. Accessed 3 May 2016 
6. Fong GT, Hammond D, Jiang Y, Li Q, Quah AC, Driezen P, Yan M. Perceptions of tobacco health warnings in China compared with picture and text-only health warnings from other countries: an experimental study. Tob Control. 2010;19(Suppl 2):169-77.

7. ITC China Project Report: Findings from the Wave 1 to 3 Surveys (2006-2009). 2012. [http://www.itcproject.org/files/TTC-China-NR-English-web-Dec142012FINAL.pdf ]. Accessed 3 May 2016.

8. Hammond D, Fong GT, McNeill A, Borland R, Cummings KM. Effectiveness of cigarette warning labels in informing smokers about the risks of smoking: findings from the International tobacco control (ITC) four Country survey. Tob Con. 2006;15(Suppl 3):iii19-25.

9. Hammond D. Health warning messages on tobacco products: a review. Tob Con. 2011;20:327-37.

10. Elton-Marshall T, Xu SS, Meng G, Quah AC, Sansone GC, Feng G, Jiang Y, Driezen P, Omar M, Awang R. The lower effectiveness of text-only health warnings in China compared to pictorial health warnings in Malaysia. Tob Con. 2015;24(Suppl 4):iv6-13.

11. Borland R, Yong HH, Wilson N, Fong GT, Hammond D, Cummings KM, Hosking W, McNeill A. How reactions to cigarette packet health warnings influence quitting: findings from the ITC four-Country survey. Addict. 2009;104(4):669-75.

12. Framework Convention on Tobacco Control. Guidelines for implementation of Article 11, "Packaging and Labelling of Tobacco Products." (Decision FCTC/COP3(10)). 2008. [http://www.who.int/fctc/guidelines/adopted/article_ 11/en/]. Accessed 3 May 2016

13. Hosking W, Borland R, Yong H-H, Fong G, Zanna M, Laux F, Thrasher J, Lee WB, Sirirassamee B, Omar M. The effects of smoking norms and attitudes on quitting intentions in Malaysia, Thailand and four Western nations: a crosscultural comparison. Psychol and Health. 2009;24(1):95-107.

14. Hofstede G, Hofstede GJ, Minkov M. Cultures and organizations: software of the mind. London: McGraw-Hill; 1991.

15. Wu C, Thompson ME, Fong GT, Li Q, Jiang Y, Yang Y, Feng G. Methods of the International tobacco control (ITC) China survey. Tob Con. 2010;19(Suppl 2):1-5.

16. ITC China Wave 4 (2011-2012) Technical Report. 2015. [http://www. itcproject.org/files/TT_China_Wave_4_Tech_Report_July_8_final.pdf ]. Accessed 3 May 2016.

17. Hitchman SC, Driezen P, Logel C, Hammond D, Fong GT. Changes in effectiveness of cigarette health warnings over time in Canada and the United States, 2002-2011. Nico Tob Res. 2013;16(5):536-43.

18. Qin Y, Wu M, Pan X, Xiang Q, Huang J, Gu Z, Shi Z, Zhou M. Reactions of Chinese adults to warning labels on cigarette packages: a survey in Jiangsu Province. BMC Public Health. 2011;11(1):133.

19. Fong GT, Hammond D, Hitchman SC. The impact of pictures on the effectiveness of tobacco warnings. Bull World Health Organ. 2009;87(8):640-3.

20. World Health Organization Western Pacific Region. University of Waterloo, ITC project and ThinkTank research Center for Health Development. Tobacco health warnings in China: evidence of effectiveness and implications for actions. Manila: World Health Organization Regional Office for the Western Pacific; 2014. http://www.wpro.who.int/china/mediacentre/releases/2014/ tobacco_health_warnings_in_china_en.pdf]. Accessed 3 May 2016

21. Azagba S, Sharaf MF. The effect of graphic cigarette warning labels on smoking behavior: evidence from the Canadian experience. Nic Tob Res. 2013;15(3):708-17.

22. Thrasher JF, Hammond D, Fong GT, Arillo-Santillán E. Smokers' reactions to cigarette package warnings with graphic imagery and with only text: a comparison between Mexico and Canada. Salud Publica Mex. 2007:49:s233-40.

23. Peters E, Romer D, Slovic P, Jamieson KH, Wharfield L, Mertz CK, et al. The impact and acceptability of Canadian-style cigarette warning labels among U.S. smokers and nonsmokers. Nicotine Tob Res. 2007 Apr;9(4):473-81.

\section{Submit your next manuscript to BioMed Central and we will help you at every step:}

- We accept pre-submission inquiries

- Our selector tool helps you to find the most relevant journal

- We provide round the clock customer support

- Convenient online submission

- Thorough peer review

- Inclusion in PubMed and all major indexing services

- Maximum visibility for your research

Submit your manuscript at www.biomedcentral.com/submit

) Biomed Central 